\title{
Models for quantitative Neuroimaging
}

\section{Prof. Francesco Orzi ${ }^{1}$}

Department of Neurological Sciences, 2nd Faculty of Medicine University of Roma, "La Sapienza"

Ospedale S. Andrea, Via di Grottarossa 1035, 00189, Rome, Italy

E-mail: francesco.orzi@uniromal.it

\section{Dr. Francesca Tari Capone}

Department of Neurological Sciences, 2nd Faculty of Medicine University of Roma, "La Sapienza" Ospedale S. Andrea, Via di Grottarossa 1035, 00189, Rome, Italy

E-mail: fra.tarielibero.it

\section{Dr. Marilena Mangiardi}

Department of Neurological Sciences, 2nd Faculty of Medicine University of Roma, "La Sapienza" Ospedale S. Andrea, Via di Grottarossa 1035, 00189, Rome, Italy

E-mail: marilenamangiardielibero.it

\begin{abstract}
A number of different tracers suitably labelled for being detected by photon emission tomography are being used for functional neuroimaging. The pictorial representation of the distribution of a given tracer within the brain tissue, however, may be meaningless if the tissue concentration of the tracer itself, for each homogenous area of the brain, is not related to the functional parameter of interest by a theoretical model. The model describes the biochemical and physiological behaviour of the tracer with respect to the functional parameter, and the variables to be measured, or to be known, in order to obtain quantization.
\end{abstract}

A few, elementary aspects of the models for measuring local rates of cerebral blood flow and glucose utilization are described.

Frontiers in Imaging Science: High Performance Nuclear Medicine Imagers for Vascular Disease Imaging (Brain and Heart)

Istituto Superiore di Sanita', Rome, Italy

13-14 November, 2006

\footnotetext{
$1 \quad$ Speaker Prof. Francesco Orzi
} 


\section{Cerebral blood flow: new measurement technique}

In the 1948 Semour Kety and colleagues developed a method for measuring cerebral blood flow and cerebral metabolic rates for oxygen and glucose in the human brain [1]. The method is based on the Fick Principle on the conservation of the matter. According to the principle, cerebral blood flow (volume per unit of mass per unit of time) is related to the time course of the artero-venous difference and to the "final" tissue concentration of a diffusible tracer. In order to measure blood flow one should, therefore, carry out tissue sampling and timed arterial and venous blood samples, for any compartment of interest. According to the original Kety and Schmidt method the brain is considered a single compartment, a sort of impenetrable black box. Data are collected by sampling blood at the entrance (any large artery vessel) and at the exit (jugular vein) of the brain. The tissue concentration of the tracer is inferred on the basis of the venous concentration at equilibrium, by assuming an instantaneous diffusion of the tracer from the blood to the brain tissue. Cerebral blood flow can thus be calculated by using the operational equation of the method. The method is a gold standard for the paucity of assumptions and elegancy of the model. The obtained measurement, however, is waited average blood flow value of the whole brain. There are no images. And all the information related to the heterogeneity of the brain is lost (fig. 1).

\section{Autoradiography techniques}

In the sixties, L. Sokoloff et al. developed quantitative autoradiography techniques and designed new theoretical models for relating the tissue concentration of a labelled tracer to blood flow. The basic principles underlying the new methods are still based of the Fick principle and on the use of a diffusible tracer, labelled with an isotope suitable for autoradiography. A general approach consists in measuring the tissue distribution of the tracer within the brain by autoradiography. The required arterial input function is obtained by sampling in a large, peripheral artery. The information on the concentration of the tracer in the venous blood, flowing out from each single homogeneous compartment inside the brain, is inferred on the basis of the measured local tissue concentrations of a diffusible tracer and on the knowledge of the tissue/blood partition coefficient of the tracer. A number of theoretical models and methods have been developed since the original formulation [2].

The tissue equilibration principle states that for any homogeneous compartment there is complete equilibrium of the tracer between tissue and venous drainage, such that any time following the introduction of the tracer in the system the venous concentration of the tracer is equal to its local tissue concentration divided by the partition coefficient constant. The principle is the support to a number of methods, including the 14C-Iodoantipyrine and quantitative autoradiography for laboratory animals, the 150-Water for PET and the Xenon method for CT. The principle, when referred to the wash out phase that follows the saturation of the system with the tracer, is also the theoretical fundament of the clearance method, widely employed with 133Xenon and gamma cameras in the past, or with SPECT.

According to the indicator distribution principle the tracer is trapped in the tissue so that the venous concentration is zero. Radioactive microspheres, of suitable size to get 
mechanically trapped into the capillary vessels, have been largely employed in experimental animals. Chemical "microspheres" (compounds with a high partition coefficient value) and SPECT are currently used in humans for evaluating blood flow values.

A requirement for assaying local cerebral blood flow is the measurement of the local tissue concentration of the tracer. The concentration values are obtained by means of a number of different techniques developed in the last few decades, ranging from autoradiography (ex vivo) for laboratory animals, to SPECT, PET or CT in vivo. The different techniques provide a pictorial representation of the difference among the brain areas in the distribution of the tracer. By doing so, they do in fact visualize relative rates of blood flow, but two fundamental steps are needed to obtain actual rates. For each area of interest in the brain, the optical density of the autoradiograph in experimental animals, or the brain radioactivity as detected by tomography in humans, has to be converted to local tissue concentrations of the tracer, on the basis of calibrated standards. The local tissue concentration value has then to be used, together with the other measurable parameters in the peripheral blood, to calculate the rates of blood flow according to the operational equation.

A main objective in measuring local rates of blood flow is often to infer on the functional activity of the brain. The brain requires huge amount of energy compared to other organs, and the energy substrates are essentially oxygen and glucose. In absence of significant energy deposit the supply of the two essential elements relies uniquely on the continuous supply by the blood. Any change of the functional activity is followed by a change in blood flow. Measurement of the glucose, or oxygen, utilization would, however, represent a better parameter of functional activity, under physiological conditions.

\section{FDG and brain metabolism}

In the seventies L. Sokoloff and collaborators, at NIH in Bethesda, developed the 14Cdeoxyglucose method for measuring local rates of glucose utilization in the rat brain.

The method exploits a fundamental approach for measuring rates of biochemical reactions, for which both the amount of reagents and products of the reaction need to be known. Glucose is not a good tracer as the metabolites would leave the brain as it is used. The 2-deoxyglucose is an analogue of glucose. Both glucose and deoxyglucose cross the blood brain barrier and enter the glycolitic pathway. Unlike glucose-6-phosphate, however, which is metabolized eventually to $\mathrm{CO} 2$ and water, deoxyglucose-6-phosphate is not a substrate for further metabolism, and it accumulates in the tissue at a rate which reflects the glucose utilization. By measuring the amount of product it is possible to infer on the rate of production and consequently on the rate of glucose utilization. An operational equation based on the theoretical model of the method defines the variable to be measured, and the kinetic constants to be known, in order to calculate the rate of glucose utilization on the basis of the tissue concentration of the accumulated deoxyglucose-6-phosphate. Deoxyglucose is labelled with $\mathrm{C} 14$, and the concentration of the isotope can be measured by autoradiography. The method is designed in such a way that the tissue concentration of the isotope at the end of the experimental time reflects essentially the concentration of the metabolic product deoxyglucose-6-phosphate. By autoradiography it is, therefore, possible to visualize in a 
quantitative way the distribution of the deoxyglucose-6-phosphate, which reflects the rate of glucose utilization (fig.2).

\section{References}

[1] Kety S. S., and Schmidt, C. F. The nitrous oxide method for the quantitative determination of cerebral blood flow in man: Theory, procedure, and normal values. J. Clin. Invest. 27: 476-483, 1948.

[2] Freygang, W. H., and Sokoloff, L. Quantitative measurements of regional circulation in the central nervous system by the use of radioactive inert gas. Adv. Biol. Med. Phys. 6: 263-279, 1958. 